\section{Path-loss and Correlation Analysis for Space and Polarization Diversity in Surface Ducts}

\author{
Ergin Dinc, Member, IEEE, Fatih Alagoz, Member, IEEE, \\ Ozgur B. Akan, Fellow, IEEE
}

\begin{abstract}
Atmospheric ducts, that are caused by the rapid decrease in the refractive index of the lower atmosphere, can trap the propagating signals. Trapping effect of atmospheric ducts provides significant received power enhancements, and enables signals to reach beyond-line-of-sight (b-LoS) distances. Although wave propagation in atmospheric ducts are well studied, there is no work that provides fading and correlation analysis for atmospheric ducts. Therefore, the main motivation of this paper is to develop a large-scale path-loss model, estimate distribution of fading, and analyze fading correlation for space and polarization diversity systems in the presence of surface ducts. Lastly, the achievable data rate results are presented to show the performance improvement with space and polarization diversity techniques.
\end{abstract}

Index Terms-Communication channels, MIMO systems, Propagation, Refraction

\section{INTRODUCTION}

Atmospheric ducts are caused by the rapid decrease in the index of refraction at the lower troposphere. Due to the rapid changes in the refractive index, the propagating signals can be refracted back to the surface, and trapped between the sea surface and atmospheric duct. The trapping effect of the atmospheric ducts provides significant received power enhancement compared to the standard atmospheric conditions. For this reason, atmospheric ducts can be utilized as a b-LoS communication medium, which is also denoted as ducting channel [1].

Ducting channel studies mostly focus on wave propagation in atmospheric ducts [1], [2]. Parabolic equation (PE) methods [3] are the most dominant technique to model ducting channel wave propagation because PE methods can model complex boundary conditions and refractivity profiles of the atmosphere. The path-loss estimates with the PE methods were experimentally validated in [4], [5]. Therefore, PE methods yield reliable results for ducting communication links.

To solve PE, specialized wave propagation softwares are developed. Firstly, AREPS [6] is the most widely used software. In [5], AREPS was utilized to estimate path-loss for a 78 $\mathrm{km}$ link, and the path-loss estimates were experimentally validated. However, we utilize PETOOL [7], which is a free and online available software operating on MATLAB. PETOOL was validated by AREPS results in [7]. Thus, PETOOL is a reliable wave propagation simulator for ducting channels. In [8], PETOOL results are utilized to develop a large-scale pathloss model for surface ducts.

E. Dinc and O. B. Akan are with the Next-generation and Wireless Communications Laboratory, Department of Electrical and Electronics Engineering, Koc University, Istanbul, 34450 Turkey (e-mail: edinc@ku.edu.tr; akan@ku.edu.tr).

F. Alagoz is with the Department of Computer Engineering, Bogazici University, Istanbul 34342, Turkey (e-mail: fatih.alagoz@boun.edu.tr).
Although there are many studies on ducting channel wave propagation, there is no study, focusing on the investigation of antenna correlations and multiple-input-multiple-output (MIMO) systems for ducting channels. Therefore, the main motivation of this paper is to analyze MIMO ducting channels in the presence of surface ducts. In this paper, we only focus on surface ducts because modified refractivity of surface ducts can be modeled with a simple bi-linear model as described in Section II.

There are three main contributions of this work. Firstly, we develop a large-scale path-loss model for surface ducts to estimate the path-loss. By using the developed large-scale path-loss model, the distribution of fading in ducting channel is predicted. Second contribution is to analyze the correlation coefficient for space and polarization diversity systems in ducting channel for the first time in the literature. Since bLoS communication systems generally operates at low signalto-noise ratio (SNR) due to high path lengths, MIMO systems have high potential to increase the achievable data rates. Thirdly, we compare the performance of different diversity techniques in terms of achievable data rates by combining the proposed path-loss and correlation analyses. This way, we aim to show that ducting channel is suitable for high data rate applications.

The remainder of the paper is organized as follows. Section II provides the review of ducting channel modeling. Section III includes the proposed large-scale and small-scale pathloss models for ducting channel. In Section IV, we provide the correlation analysis for space and polarization diversity. Section V includes the capacity calculations with the proposed models. The achievable data rate results are presented in Section VI. Lastly, conclusions are presented in Section VII.

\section{Channel Modeling in Ducting Channels}

This section includes the propagation and atmospheric modeling for ducting channels.

\section{A. Parabolic Equation Method}

PE was originally developed in [9], [10], and utilizes paraxial approximation to the Helmholtz wave equation. PE methods became the dominant technique to model wave propagation in atmospheric ducts after the derivation of a computationally efficient solution based on Split-Step Fourier (SSF) transform [10]. PE is especially advantageous to model complex boundary conditions and refractivity variations of the lower atmosphere [3]. PE methods are mostly utilized in ducting channel wave propagation analysis because wave propagation in ducting channel depends on multiple parameters: transmitter height, receiver height, duct height, duct strength, carrier frequency, polarization and surface conditions.

For 2D narrow angle forward scatter waves, PE is represented as [2]

$$
\frac{\partial u(x, z)}{\partial x}=\frac{i}{2 k} \frac{\partial^{2} u(x, z)}{\partial z^{2}}+\frac{i k}{2}\left(m^{2}(x, z)-1\right) u(x, z),
$$

where $x$ is the range in $\mathrm{m}, z$ is the height in $\mathrm{m}, k=2 \pi / \lambda$ is the wave number, $\lambda$ is the wavelength, and $u(x, z)$ is the reduced 
function. The modified refractivity index is $m=1+M 10^{-6}$, where $M$ is the modified refractivity. Horizontal and vertical polarization can be modeled by (1) with appropriate boundary conditions.

In this paper, PETOOL [7] is utilized to solve (1), and the path-loss $(P L)$ is calculated as [3]

$$
P L=20 \log (4 \pi /|u(x, z)|)+10 \log (d)-30 \log (\lambda),
$$

where $d$ is the range in m. In Section III, PETOOL simulation results are utilized to develop large-scale path-loss model and analyze small-scale path-loss characteristics.

\section{B. Atmospheric Modeling}

In PE methods, atmospheric conditions are considered via modified refractivity, which is represented as

$$
M=N+\left(h / R_{0}\right) \times 10^{6} \text { M-units, }
$$

where $h$ is the height in $\mathrm{km}$ and $R_{0}$ is the earth radius in $\mathrm{km}$. Refractivity is represented with $N=(n-1) \times 10^{6} \mathrm{~N}$-units, where $n$ is the atmospheric refractive index.

Trapping layer of atmospheric ducts is associated with negative modified refractivity gradient as in Figure 1. This condition causes propagating signals to be refracted back to the sea surface, and these signals are trapped between the sea surface and atmospheric duct. This way, trapped signals can propagate over-the-horizon with lower path-loss compared to the standard atmospheric conditions. This effect makes ducting channel a promising candidate for b-LoS communications, especially in coastal and maritime environments [1].

Atmospheric ducts can be classified according to their formation process and properties: evaporation ducts, surfacebased ducts, elevated ducts, and surface ducts [1]. The occurrence probability of surface and evaporation ducts are significantly higher than surface-based ducts and elevated ducts. Therefore, surface and evaporation ducts are more suitable for b-LoS communications. Surface ducts can be modeled with a bi-linear curve and depend on two parameters: duct height $(\delta)$ and duct strength $(\Delta M)$ as presented in Figure 1(a). Duct height $(\delta)$ represents the height of the trapping layer. Duct strength is the change in the modified refractivity from surface to trapping layer. On the other hand, evaporation ducts can be modeled with a logarithmic curve as in Figure 1(b). Ray tracing analysis for the bi-linear model is computationally efficient to estimate angle-of-arrivals and delay spreads of the ducting channels. For this reason, evaporation ducts are also approximated by a bi-linear curve in the propagation analysis [11]. Thus, we only focus on surface ducts in the remainder of the paper.

Surface ducts are modeled with duct height and duct strength. Modified refractivity above the duct height is modeled as the standard atmospheric conditions (Modified refractivity increases with $118 \mathrm{M}$-units $/ \mathrm{km}$.). According to the experimental studies [12], [13], the surface duct formation has up to $80 \%$ probability of occurrence, and even in winter conditions with low evaporation rates this probability is above $60 \%$. Due to the high formation probabilities, trapping effect of surface ducts can be utilized as a b-LoS communication medium.

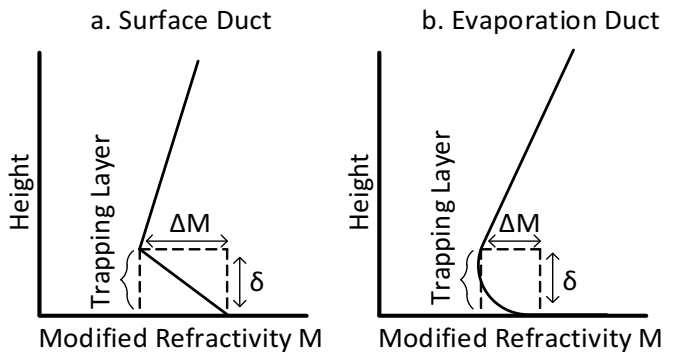

Fig. 1: Modified refractivity profiles for (a) surface duct, and (b) evaporation duct.

\section{LARGE-SCALE AND SMALl-SCALE PATH-LOSS MODEl FOR SURFACE DUCTS}

This section provides the large-scale path-loss model for surface ducts and the distribution of the small-scale path-loss. To this end, we develop a large-scale path-loss model for the surface ducts based on regression analysis to PETOOL results as in [8]. Then, deviations from our large-scale path-loss model are denoted as the small-scale path-loss, which is also known as fading.

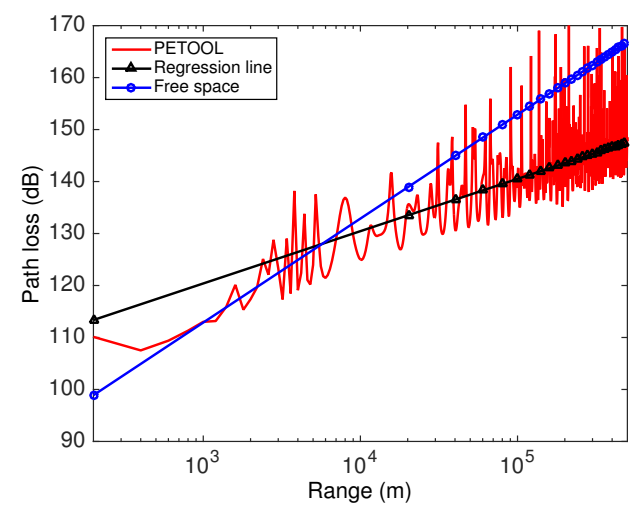

Fig. 2: Path-loss results with PETOOL and regression model.

Ducting channel communication is especially promising in places, where direct LoS communication is not available or expensive [5]. Therefore, ducting communication studies focus on b-LoS ranges. For a wireless channel, the generic largescale path-loss equation is given as [14]

$$
P L_{d B}=A+10 \gamma \log d / d_{0}+X,
$$

where $X$ is the small-scale fading, $\gamma$ is the path-loss exponent, $d$ is the range in $\mathrm{m}, A$ is the path-loss in $\mathrm{dB}$ at $d_{0}$, and $d_{0}$, as defined in (5), is the fixed range in $\mathrm{m}$ where b-LoS is observed [15].

$$
d_{0}=\left(4.12 \sqrt{h_{t}}+4.12 \sqrt{h_{r}}\right) \times 10^{3} \mathrm{~m},
$$

where $h_{t}$ and $h_{r}$ are the transmitter and receiver heights in $\mathrm{m}$, respectively.

For the large-scale path-loss model, we first generate pathloss results for ducting channel with PETOOL for the following parameters: frequency $10 \mathrm{GHz}$, transmitter height $20 \mathrm{~m}$, receiver height $27 \mathrm{~m}$, horizontal polarization, duct height 40 $\mathrm{m}$, duct strength $20 \mathrm{M}$-units. Figure 2 includes the path-loss 


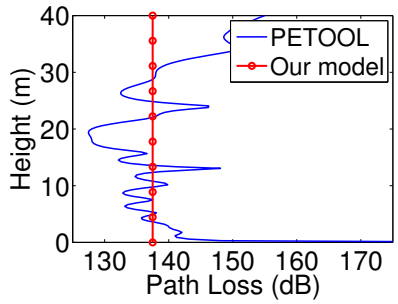

(a) $50 \mathrm{~km}$.

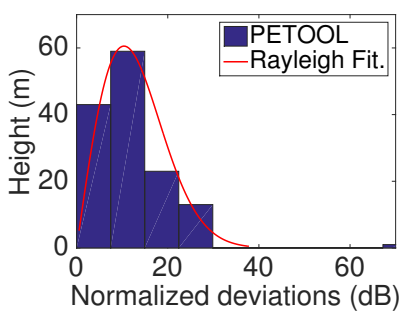

(d) $50 \mathrm{~km}$.

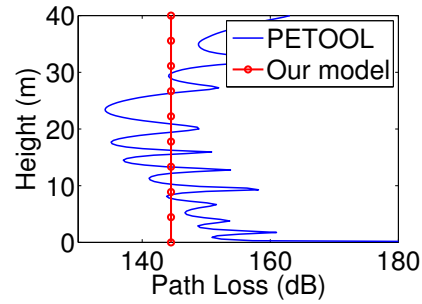

(b) $250 \mathrm{~km}$.

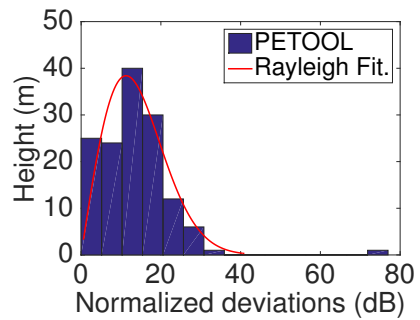

(e) $250 \mathrm{~km}$.

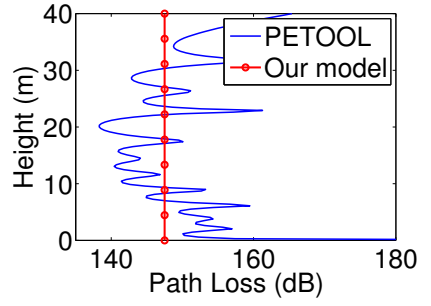

(c) $500 \mathrm{~km}$.

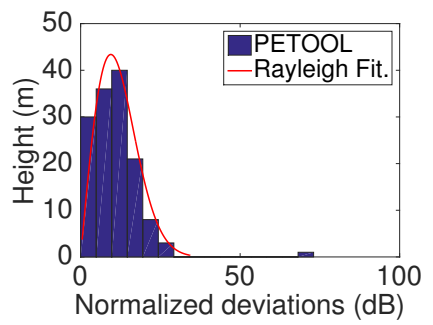

(f) $500 \mathrm{~km}$.

Fig. 3: (a-c) The comparison of PETOOL and our model, (d-f) histograms of the deviations with Rayleigh distribution fitting.

vs. range for fixed receiver height. Then, we apply regression analysis to fit the path-loss results presented in Figure 2 to (4) in the least square error sense as in [8], [16]. In this way, $A$ and $\gamma$ parameters in (4) are found as $A=136 \mathrm{~dB}$ and $\gamma=1.03$. Figure 2 also includes the regression based model (straight line in Figure 2) and the free-space path-loss. As noticed, the surface duct can provide $20-30 \mathrm{~dB}$ lower path-loss compared to the free space conditions as also reported in [4].

Figure 3(a-c) present the path-loss estimates of PETOOL and the proposed regression based path-loss estimates for different ranges. As noticed, the level of the large-scale path-loss can be estimated with the developed model. The deviations from the regression model can be assumed as fading in the ducting channel. Figure 3(d-f) present the normalized distribution of the fading in the ducting channel for different ranges along with the Rayleigh distribution fitting. The Rayleigh distribution fitting in Figure 3(d-f) has standard deviation between $8-9 \mathrm{~dB}$. To justify the Rayleigh distribution assumption, we utilize the two-sample Kolmogorov-Smirnov test [17] on MATLAB. According to the test, the presented histograms in Figure 3(d-f) can be fitted with the Rayleigh distribution within $85-90 \%$ confidence level. Hence, the ducting channel with the surface ducts can be modeled as a Rayleigh fading channel.

\section{Antenna Correlations for Space And POLARIZATION DIVERSITY}

Ducting b-LoS communications suffer from high path-losses due to high communication ranges. Therefore, MIMO techniques have high potential to improve the achievable data rates. For this reason, we analyze the fading correlation for space and polarization diversity systems for ducting communication links.

\section{A. Space Diversity}

Space diversity can be achieved with horizontal or vertical placement. In this paper, only vertical space diversity is considered because vertical space diversity becomes uncorrelated with a few meters separation. To calculate the space correlations, we generate path-loss results for vertically spaced transmitters, and calculate the correlation coefficient among the vertical variations of the path-loss at the desired range. Figure 4 shows the antenna correlation vs. vertical spacing for different carrier frequencies and different duct heights with transmitter height $27 \mathrm{~m}$ and duct strength rate $1 \mathrm{M}$ units/m (Duct strength is equal to duct strength rate times duct height.). As noticed, $1 \mathrm{~m}$ separation provides uncorrelated antennas (correlation coefficient close to 0 ) for $15 \mathrm{GHz}$. For $10 \mathrm{GHz}, 1-2 \mathrm{~m}$ spacing yields low correlation values. The required spacing decreases as the carrier frequency increases. We conclude that the vertical space diversity is promising for ducting channels.

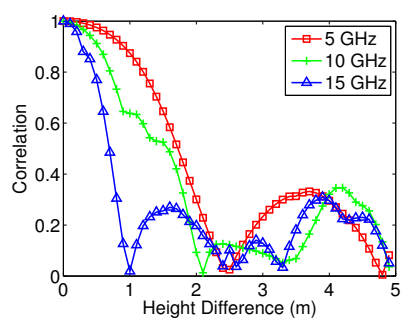

(a) $\delta=20 \mathrm{~m}$.

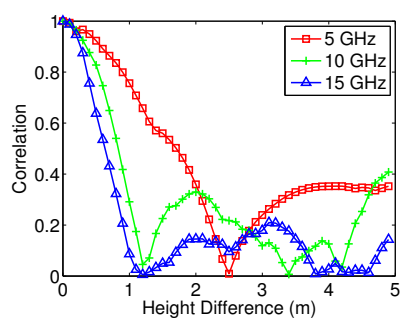

(b) $\delta=30 \mathrm{~m}$.

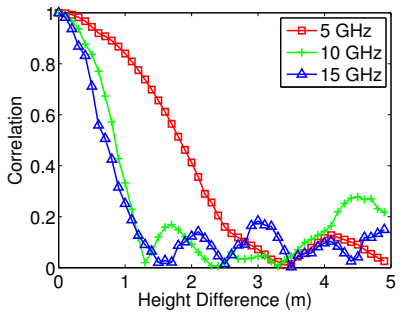

(c) $\delta=40 \mathrm{~m}$.

Fig. 4: Antenna correlation for vertical diversity with different duct heights. 


\section{B. Polarization Diversity}

Polarization diversity is an advantageous method in wireless systems to provide higher data rates with lower cost [18], [19] because polarization diversity can be implemented with dualpolarized antennas. This way, polarization diversity does not require an additional antenna. In order to utilize polarization diversity, the correlation among the vertical and horizontal polarization should be lower than a certain threshold $(\approx 0.5)$. To this end, we analyze the correlation between horizontal and vertical polarization in b-LoS ducting links.

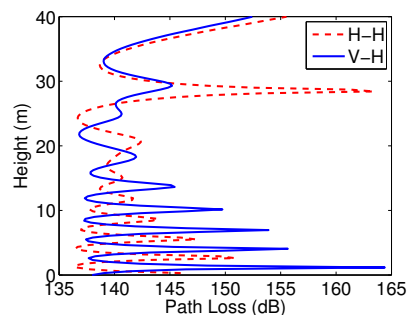

(a) Path-loss results.

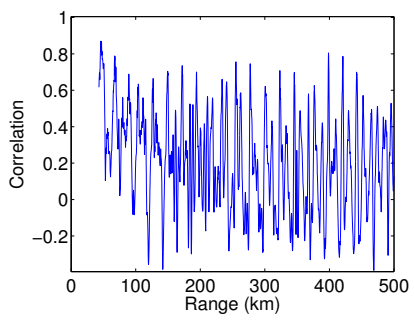

(b) Correlation vs. range.

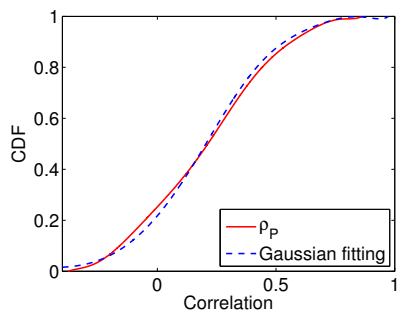

(c) $\mathrm{CDF}$ of correlations.

Fig. 5: Fading correlation for polarization diversity.

We also utilize PETOOL simulations to analyze the correlation in polarization diversity. For this case, we simulate transmitters with horizontal and vertical polarization. We use the following channel parameters: transmitter/receiver heights $27 \mathrm{~m}$, horizontal polarization in the receiver, sea surface conditions, duct height $40 \mathrm{~m}$ and duct strength $10 \mathrm{M}$-units. Figure 5(a) presents the vertical path-loss results at $100 \mathrm{~km}$ range, where $\mathrm{H}$ and $\mathrm{V}$ represent horizontal and vertical polarization, respectively. As noticed, the fading in different transmitter polarization shows significant difference.

Fading correlation among polarization $\left(\rho_{P}\right)$ with respect to range is presented in Figure 5(b). Figure 5(c) shows the cumulative distribution function $(\mathrm{CDF})$ of the correlation among the polarization. As noticed, $80 \%$ of the time the correlation is below 0.5 . Although the standard deviation of the fading correlation is less between $50-100 \mathrm{~km}$ range, the overall fading correlation can be approximated by a Gaussian distribution with mean 0.2 and standard deviation 0.27 because ducting b-LoS communication is aimed to be utilized at b-LoS ranges, which are often higher than $100 \mathrm{~km}$. As in Section III, we use the two-sample Kolmogorov-Smirnov test [17] to justify the fitted distribution in Figure 5(c). The test yields that the fitted distribution has $95 \%$ confidence level. Since MIMO systems can achieve desired capacity gains below 0.5 correlation, polarization diversity is a promising method for ducting channels. The occurrence rate of surface ducts mostly varies between $70-80 \%$ depending on the season. Therefore, the polarization diversity will be able to provide the expected gains up to $64 \%$ of the time. On the other hand, the correlation among horizontal and vertical polarization at the receiver is close to one according to our simulations. In conclusion, polarization diversity is promising for ducting links as a transmitter diversity technique.

\section{Channel Capacity in Surface Duct Links}

In this section, we introduce capacity calculations to calculate achievable data rates for ducting channel by utilizing the proposed regression model and correlation results in Section III and IV, respectively. Since the previous ray-tracing analysis suggests that b-LoS ducting channel has delay spreads in the order of nanoseconds [8], [20], [21], we utilize a narrowband fading model for ducting channel with surface ducts. Therefore, multi-path components are not resolvable within the desired bandwidth limits $(<40 \mathrm{MHz})$. As described in Section III, the ducting channel is assumed to have Rayleigh fading. As a result, we utilize flat Rayleigh fading channel model.

Suppose $N_{T}$ transmitters and $N_{R}$ receivers use the channel. We can model the channel as

$$
\mathbf{y}=\mathbf{H x}+\mathbf{n},
$$

where $\mathbf{H}_{N_{R} \times N_{T}}$ is the channel gain matrix, $\mathbf{x}_{N_{T} \times 1}$ and $\mathbf{y}_{N_{R} \times 1}$ are the transmit and receive vectors, and $\mathbf{n}_{N_{R} \times 1}$ is the noise vector. Since $\mathbf{H}$ contains the effect of both large-scale pathloss and small-scale path-loss, we utilize channel matrix normalization to separate these two effects. Thus, the normalized channel gain matrix $\tilde{\mathbf{H}}$ is represented as [22]

$$
\tilde{\mathbf{H}}=\frac{\mathbf{H}}{g} \text {. }
$$

Here, $g$ is calculated as

$$
g=\sqrt{\frac{1}{N_{T} N_{R}}\|\mathbf{H}\|_{F}^{2}},
$$

where $\|.\|_{F}$ is the Frobenius norm.

With the channel matrix normalization, the average receive power in Watts is given by [22], [23]

$$
P r_{a v}=g^{2} P_{t}=P_{t} G_{t} G_{r}\left(P L_{L}\right)
$$

where $P_{t}$ is the transmit power in $\mathrm{W}, G_{t}$ and $G_{r}$ are the transmitter and receiver antenna gains, respectively. Lastly, $P L_{L}$ is the large-scale path-loss, which is directly calculated with the developed large-scale path-loss model $\left(P L_{L}\right)_{d B}=$ $A+10 \gamma \log d / d_{0} \mathrm{~dB}$ as in (4), where $A$ and $\gamma$ are calculated as in Section III. In this way, $g^{2} P_{t}$ will be directly calculated by using the developed large-scale path-loss model by (9) for the given channel parameters: $P_{t}, G_{t}, G_{r}$. In this paper, we assume that large parabolic reflector antennas are utilized to provide high antenna gains. However, high antenna gains can be provided via planar antenna arrays as proposed in [24].

The effects of small-scale path-loss are modeled with $\tilde{\mathbf{H}}$. The normalized channel gain matrix can be represented as [25]

$$
\tilde{\mathbf{H}}=\left(\mathbf{R}^{R X}\right)^{1 / 2} \mathbf{G}\left(\mathbf{R}^{T X}\right)^{1 / 2},
$$


where $\mathbf{G}_{N_{R} \times N_{T}}$ is the complex Gaussian independent and identically distributed channel matrix. The root of the receiver antenna correlation matrix $\left(\left(\mathbf{R}^{R X}\right)^{1 / 2}\right)$ can be computed with the Cholesky factorization. In the same way, $\left(\mathbf{R}^{T X}\right)^{1 / 2}$ is the root of the transmitter antenna correlations $\left(\mathbf{R}^{T X}\right)_{N_{T} \times N_{T}}$. The correlation matrices depend on the type of diversity. In this study, we assume that the channel is symmetrical for vertical space diversity for simplicity because the modeled surface duct is range independent and same number of antennas are utilized at both ends with the same orientations. Thus, the calculated correlation values are assumed to be valid for both transmitter and receiver in the vertical space diversity.

For space diversity: The transmitter correlation matrix for $N_{T} \times N_{R}$ space diversity is shown as

$$
\mathbf{R}^{T X}=\left[\begin{array}{cccc}
1 & \rho_{1,2}^{S} & \ldots & \rho_{1, N_{T}}^{S} \\
\rho_{2,1}^{S} & 1 & \ddots & \vdots \\
\vdots & \ddots & \ddots & \vdots \\
\rho_{N_{T}, 1}^{S} & \cdots & \cdots & 1
\end{array}\right],
$$

where $\rho_{i, j}^{S}$ is the correlation between antenna $i$ and $j$ and, they are determined by using the analysis in Figure 4 according to the channel parameters. Note that, the $N_{R} \times N_{R}$ receiver correlation matrix has also the same structure as (11).

For polarization diversity: The polarization diversity is effective only for transmitter side since polarization has high correlation at the receiver side. Therefore, we assume that all receivers are horizontally polarized. For a dual-polarized antenna, the transmitter correlation matrix is given as

$$
\mathbf{R}_{P}^{T X}=\left[\begin{array}{cc}
1 & \rho_{1,2}^{P} \\
\rho_{2,1}^{P} & 1
\end{array}\right],
$$

where $\rho_{1,2}^{P}=\rho_{2,1}^{P}$ is the correlation between horizontal and vertical polarization. As shown in Figure 5, the correlation between polarization can be modeled with a Gaussian random variable with mean 0.2 and standard deviation 0.27 as described in Section IV-B.

For space-polarization diversity: We also consider spatially separated dual-polarized transmitters. The transmitter correlation matrix for $N_{T}$ number of dual-polarized transmitters is given as

$$
\left(\mathbf{R}_{T X}^{S P}\right)_{2 N_{T} \times 2 N_{T}}=\left[\begin{array}{ccc}
{\left[\mathbf{R}_{P}^{T X}\right]_{1,1}} & \ldots & \rho_{1, N}^{S}\left[\mathbf{R}_{P}^{T X}\right]_{1, N_{T}} \\
\vdots & \ddots & \vdots \\
\rho_{N, 1}^{S}\left[\mathbf{R}_{P}^{T X}\right]_{N_{T}, 1} & \ldots & {\left[\mathbf{R}_{P}^{T X}\right]_{N_{T}, N_{T}}}
\end{array}\right]
$$

where the correlation matrices for dual-polarized antennas $\left[\left(\mathbf{R}_{P}\right)_{2 \times 2}\right]_{\left\{1: N_{T}, 1: N_{T}\right\}}$ are determined randomly as in (12). Since dual-polarized antennas are utilized at the transmitter side, the total number of transmitters becomes $2 N_{T}$ for $N_{T}$ number of dual-polarized transmitters.

By using the matrix normalization in (9), the resulting channel capacity is calculated as

$$
\begin{aligned}
C & =\log _{2} \operatorname{det}\left(\mathbf{I}_{N_{R}}+\frac{P r_{a v}}{N_{T} \sigma_{n}^{2}} \tilde{\mathbf{H}} \tilde{\mathbf{H}}^{H}\right), \\
& =\log _{2} \operatorname{det}\left(\mathbf{I}_{N_{R}}+\frac{g^{2} P_{t}}{N_{T} \sigma_{n}^{2}} \tilde{\mathbf{H}} \tilde{\mathbf{H}}^{H}\right),
\end{aligned}
$$

where $\sigma_{n}^{2}$ is the noise in the system, $N_{T}$ is the total number of transmitters, $\mathbf{I}_{N_{R}}$ is $N_{R} \times N_{R}$ identity matrix, and $g$ is utilized in the channel matrix normalization in (8). Since there is a power amplifier at the receiver, the amplified noise at the receiver can be found as

$$
\sigma_{n}^{2}=k\left(T_{a}+T_{e}\right) B N_{F} G_{a},
$$

where $k$ is the Boltzman's constant, $G_{a}$ is the amplifier gain, $T_{a}$ is antenna noise temperature, and $T_{e}$ is effective noise temperature, which is give as $N_{F}-1=T_{e} / T_{a}$ and $N_{F}$ is the amplifier noise figure.

By denoting $\gamma=\frac{g^{2} P_{t}}{N_{T} \sigma_{n}^{2}}$, the capacity is simplified as

$$
C=\sum_{i=1}^{N_{\min }} \log _{2}\left(1+\gamma \lambda_{i}\right)
$$

where $N_{\min }=\min \left(N_{T}, N_{R}\right), \lambda_{i}$ 's are the nonzero eigenvalues of the Wishart matrix $\left(\tilde{\mathbf{H}} \tilde{\mathbf{H}}^{H}\right.$ for $N_{R} \leq N_{T}$, or $\tilde{\mathbf{H}}^{H} \tilde{\mathbf{H}}$ for $\left.N_{R}>N_{T}\right)$.

\section{Performance Evaluations}

In this section, we provide the data rate results for the diversity techniques. We utilize PETOOL to determine the parameters for our large-scale path-loss model as in Section III. Channel parameters that are utilized in the simulations are listed in Table I. We perform 10000 iterations on MATLAB for the random channel gain matrix (10) and random polarization correlation coefficient (12).

TABLE I: Ducting channel communication parameters.

\begin{tabular}{lcc}
\hline Parameter & Value & Unit \\
\hline Range $(d)$ & 300 & $\mathrm{~km}$ \\
\hline Carrier frequency $(f)$ & 10 & $\mathrm{GHz}$ \\
\hline Transmit power $\left(P_{t}\right)$ & 1 & $\mathrm{~W}$ \\
\hline TX\&RX height & 27 & $\mathrm{~m}$ \\
\hline Bandwidth $(B)$ & 20 & $\mathrm{MHz}$ \\
\hline Antenna gains $\left(G_{t, r}\right)$ & 30 & $\mathrm{dBi}$ \\
\hline Amplifier gain $\left(G_{a}\right)$ & 50 & $\mathrm{~dB}$ \\
\hline Amplifier noise figure $\left(N_{F}\right)$ & 1.32 & $\mathrm{~dB}$ \\
\hline Vertical spacing $\left(d_{v}\right)$ & 1 & $\mathrm{~m}$ \\
\hline Spatial correlation $\left(\rho^{S}\right)$ & 0.5 & - \\
\hline
\end{tabular}

Figure 6 shows the capacity results for single-input-singleoutput (SISO), space diversity, polarization diversity and space-polarization diversity in ducting channels with $40 \mathrm{~m}$ surface duct and $10 \mathrm{M}$-units duct strength. Note that, the polarization diversity significantly improves the data rates of the link as in Figure 6. Since dual-polarized transmitters can be mounted on a single antenna feeder, the cost of polarization diversity is significantly lower compared to the space diversity. However, the space diversity can provide higher diversity gains with additional antennas at both sides. By utilizing 2 dualpolarized transmitters and 2 horizontally polarized receivers, the achievable data rates can be significantly improved. Therefore, polarization diversity and vertical space diversity are promising methods for ducting channel communication systems to provide higher data rate levels. 


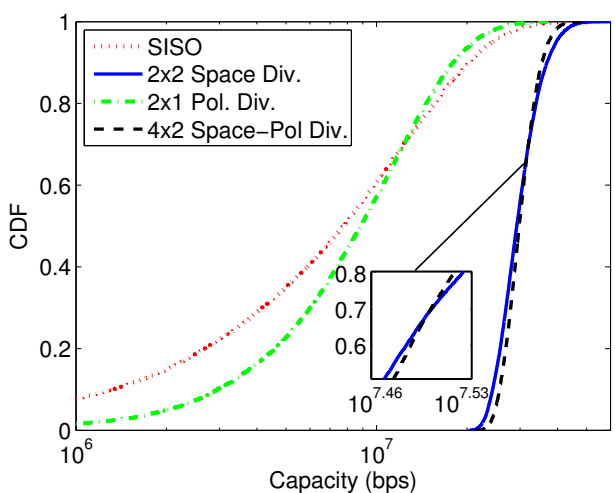

Fig. 6: Ducting channel capacity with diversity techniques.

\section{CONCLUSIONS}

In this paper, we develop a large-scale path-loss model for the ducting channel in the presence of atmospheric ducts. In addition, the distribution of fading and correlation between antennas for space and polarization diversity are analyzed. According to our results, polarization diversity is an effective diversity technique for ducting channels. In addition, $2 \times 2$ ducting channel communication can provide more than 22 Mbps at $90 \%$ reliability, thus modern high data rate applications can utilize ducting effects especially in maritime and coastal areas. In conclusion, this work reveals that ducting channel is suitable for high data rate communication applications.

\section{REFERENCES}

[1] E. Dinc, O. B. Akan, "Beyond-Line-of-Sight Communications with Ducting Layer," IEEE Commun. Mag., vol. 52, pp. 37-43, Oct. 2014.

[2] I. Sirkova, "Brief review on PE method application to propagation channel modeling in sea environment" Central European Journal of Engineering, vol. 2, issue 1, pp. 19-38, 2012.

[3] M. Levy, Parabolic Equation Methods for Electromagnetic Wave Propagation. London, U.K.: Institution of Electrical Engineers, 2000.

[4] H. V. Hitney, J. H. Richter, R. A. Pappert, K. D. Anderson, G. B. Jr. Baumgartner, "Tropospheric radio propagation assessment," Proceedings of the IEEE, vol. 73, no. 2, pp. 265-283, Feb. 1985.

[5] G. S. Woods, A. Ruxton, C. Huddlestone-Holmes, G. Gigan, "HighCapacity, Long-Range, Over Ocean Microwave Link Using the Evaporation Duct," IEEE J. Ocean. Eng., vol. 34, no. 3, July 2009.

[6] Users Manual for Advanced Refractive Effects Prediction System (AREPS). SD, CA: Space and Naval Warfare Systems Center, 2004.

[7] O. Ozgun, G. Apaydin, M. Kuzuoglu, L. Sevgi, "PETOOL: MATLABbased one-way and two-way split-step parabolic equation tool for radiowave propagation over variable terrain", Computer Physics Соттиnications, vol. 182, issue 12, pp. 2638-2654, Dec 2011.

[8] E. Dinc, O. B. Akan, "Channel Model for the Surface Ducts: Large-scale Path-Loss, Delay Spread, and AOA," to appear in IEEE Transactions on Antennas and Propagation, vol. 63, no. 6, pp. 2728-2738, June 2015.

[9] V. A. Fock, "Solution of the problem of propagation of electromagnetic waves along the earths surface by method of parabolic equations," J. Phys. USSR, vol. 10, pp. 1335, 1946.

[10] R. H. Hardin and F. D. Tappert, "Application of the split-step Fourier method to the numerical solution of nonlinear and variable coefficient wave equations, SIAM Rev., vol. 15, pp. 423, 1973.

[11] V. K. Ivanov, V. N. Shalyapin , Yu. V. Levadny, "Microwave scattering by tropospheric fluctuations in an evaporation duct," Radiophysics and Quantum Electronics, vol. 52, iss. 4, pp. 277-286, April 2009.

[12] S. S. Mentes, Z. Kaymaz, "Investigation of Surface Duct Conditions over Istanbul, Turkey," Journal of Applied Meteorology and Climatology, vol. 46, pp 318-338, 2007.
[13] S. M. Babin "Surface Duct Height Distributions for Wallops Island, Virginia, 1985-1994," Journal of Applied Meteorology and Climatology, vol. 35, pp. 8693, 1996.

[14] A. Goldsmith, "Wireless Communications"2, Cambridge University Press, 2005.

[15] C. Levis, J. T. Johnson, F. L. Teixeira, "Radiowave Propagation: Physics and Applications," WILEY, June 2010, ch. 7.5, pp. 149-151.

[16] V. Erceg, L. J. Greenstein, S. Y. Tjandra, S. R. Parkoff, A. Gupta, B. Kulic, A. A. Julius, R. Bianchi, "An empirically based path loss model for wireless channels in suburban environments," IEEE Journal on Selected Areas in Communications, vol. 17, no. 7, pp. 1205-1211, Jul 1999.

[17] I. T. Young, "Proof without prejudice: use of the Kolmogorov-Smirnov test for the analysis of histograms from flow systems and other sources," J. Histochem. Cytochem., vol. 25, no. 7 pp. 935-941, 1977.

[18] L. Dong, H. Choo, R. W. Heath, H. Ling,"Simulation of MIMO channel capacity with antenna polarization diversity," IEEE Transactions on Wireless Communications, vol. 4, no. 4, pp. 1869-1873, July 2005.

[19] M. R. Andrews, P. P. Mitra, R. deCarvalho, "Tripling the capacity of wireless communications using electromagnetic polarization," Nature, pp. 316-318, Jan. 2001.

[20] S. A. Parl, "Characterization of multipath parameters for line-of-sight microwave propagation," IEEE Transactions on Antennas and Propagation, vol. 31, no. 6, pp. 938-948, Nov. 1983.

[21] L. Pickering, J. K. DeRosa, "Refractive Multipath Model for Line-ofSight Microwave Relay Links," IEEE Transactions on Communications, vol. 27, no. 8, pp. 1174-1182, Aug. 1979.

[22] P. Kyritsi, D. C. Cox, "Effect of element polarization on the capacity of a MIMO system," in Proc. IEEE WCNC 2002, vol. 2, pp. 892-896, Mar. 2002.

[23] Kai Yu, M. Bengtsson, B. Ottersten, D. McNamara, P. Karlsson, M. Beach, "Second order statistics of NLOS indoor MIMO channels based on $5.2 \mathrm{GHz}$ measurements," in Proc. IEEE GLOBECOM, vol. 1, 2001.

[24] E. Dinc, O. B. Akan, "Limited Feedback Multi-stage Beam-forming Method for Beyond-Line-of-Sight Ducting Channels," to appear in Proc. IEEE MILCOM 2015, Tampa, FL, USA, October 2015.

[25] D. Gesbert, H. Bolcskei, D. Gore, A. Paulraj, "MIMO wireless channels: capacity and performance prediction," in Proc. IEEE GLOBECOM, vol. 2, pp. 1083-1088, vol. 2, 2000. 